\title{
Cecal Volvulus following a Right Nephrectomy for Wilms' Tumor: Should We Need to Close the Lateral Peritoneum?
}

\author{
Mauricio Gonzalez-Urquijo ${ }^{1}$ Christian Ovalle-Chao ${ }^{1}$ Eduardo Flores-Villalba ${ }^{1}$ \\ Ulises de Jesus Garza-Luna ${ }^{2}$ Jose Humberto Velazco-De La Garza ${ }^{1}$ Ulises Garza-Serna ${ }^{1}$
}

${ }^{1}$ Department of Surgery, Escuela de Medicina, Tecnologico de
Monterrey, Monterrey, Nuevo Leon, Mexico
${ }^{2}$ Department of Paediatric Surgery, Universidad Autonoma de Nuevo
Leon, San Nicolas de los Garza, Nuevo Leon, Mexico

\author{
Address for correspondence Ulises Garza-Serna, MD, FEBPS, Escuela \\ de Medicina, Tecnologico de Monterrey, Dr. Ignacio Morones Prieto O \\ 3000. Monterrey, Nuevo Leon 64849, Mexico \\ (e-mail: ugarza@itesm.mx).
}

Eur J Pediatr Surg Rep 2018;6:e1-e3.

\author{
Abstract \\ Keywords \\ - cecal volvulus \\ - lateral peritoneum \\ - nephrectomy \\ - Wilms' tumor
}

Wilms' tumor (WT) accounts for $90 \%$ of all pediatric renal malignant tumors. The most common postoperative complication based on the National Wilms' Tumor Study is small bowel obstruction. We report on a 2-year-old girl with postoperative bowel obstruction following a right nephrectomy for WT. The patient was reintervened 48 hours after surgery and a cecal volvulus was found. Here, we will describe possible causes of this postoperative complication and discuss management.

\section{New Insights and the Importance for the Pediatric Surgeon}

When performing a nephrectomy it might be important to consider closing the lateral peritoneum as it might play a role in avoiding possible post-operative cecal volvulus.

\section{Introduction}

Wilms' tumor (WT) is the most common childhood malignant renal tumor, it represents almost $90 \%$ of pediatric renal tumors and $7 \%$ of all childhood malignancies, occurring mainly in patients younger than 5 years of age and it has a 5 -year survival rate of $85 \%{ }^{1,2}$

Surgical complications based on the National Wilms' Tumor Study (NWTS) protocol with an upfront primary nephrectomy has significantly decreased over the past decade with postoperative bowel obstruction of the small intestine being the most common. ${ }^{3}$

Large bowel volvulus is a rare cause of bowel obstruction occurring mainly in pediatric patients, with the cecum being the most common location in some series and it represents less than $1 \%$ of all intestinal obstructions in children. ${ }^{4-7}$ Although postoperative cecal volvulus following a nephrectomy in adult patients $^{5,8-10}$ have been described previously, to our knowledge, there are no reported cases of a cecal volvulus following a nephrectomy in children and more specifically after a WT resection.

We present the case of a 15-month-old female patient with a cecal volvulus following a right nephrectomy of a WT.

\section{Case Report}

A 15-month-old female patient was brought to our teaching hospital and was admitted through the emergency room following a nonpainful abdominal distention and a right abdominal mass. An ultrasound confirmed the presence of a right renal mass, and a computed tomography (CT) scan showed an $8 \mathrm{~cm} \times 7 \mathrm{~cm}$ right renal tumor; no thrombosis of the renal or inferior vena cava was observed ( - Fig. 1 ). A chest CT scan showed no evidence of pulmonary metastasis. The patient was then scheduled for a right nephrectomy. received

August 11, 2017

accepted after revision

November 2, 2017

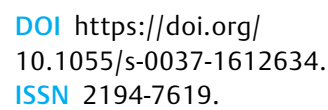

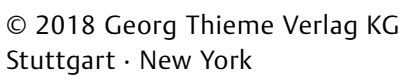

License terms

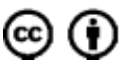



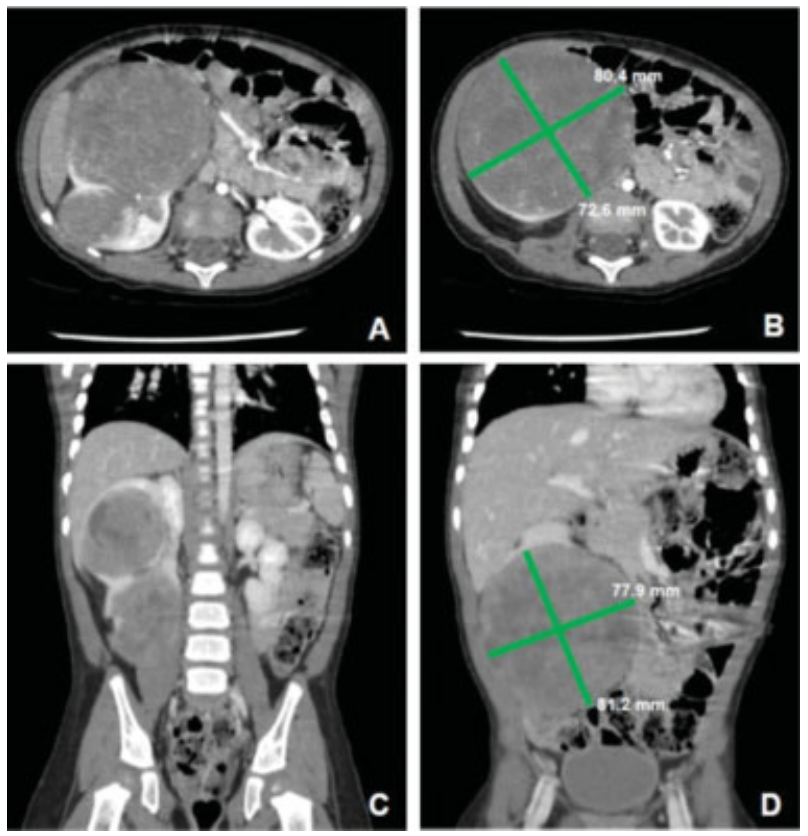

Fig. 1 Abdominal computed tomography scan with intravenous contrast. (A, B) (Superior to inferior cuts) axial view; (C, D) (posterior to anterior cuts) coronal view; $8 \times 7 \mathrm{~cm}$ right kidney tumor.

For a better longitudinal exposure of the vena cava, we entered through an incision in the midline, immediately displacing the cecum and the right colon medially. The right lateral peritoneum was divided to allow access to the renal tumor and finally the right kidney was removed along with a para-aortic lymphadenectomy. The right colon and the small bowel were left in place inside the abdominal cavity during the entire procedure, and we did not close the lateral peritoneum before closing the abdominal wall. Forty-eight hours after surgery, the patient showed abdominal distention and one episode of bilious vomiting. An abdominal X-ray showed the presence of fixed right lower abdominal air fluid levels and showed small bowel dilatation ( - Fig. 2). The patient was taken to the operating room for an exploratory laparotomy performed through the previous incision. The small bowel was intact, and a cecal volvulus was found (-Fig. 3). A detorsion was performed showing a viable bowel and the lateral peritoneum was closed. The patient had an uneventful recovery and was discharged on postoperative day 5. Pathology confirmed a multifocal right renal stage I WT.

\section{Discussion}

Based on the NWTS protocol, nephrectomy is the primary upfront treatment for WT. Preoperative chemotherapy is reserved for patients who have a solitary kidney, a horseshoe kidney, thrombosis of the inferior vena cava above the level of the hepatic veins, or if the child presents with respiratory distress due to extensive pulmonary metastases. ${ }^{11-13}$ Based on the CT scan, our patient did not meet the criteria for preoperative chemotherapy according to the NWTS protocols; therefore, we decided to perform a nephrectomy.

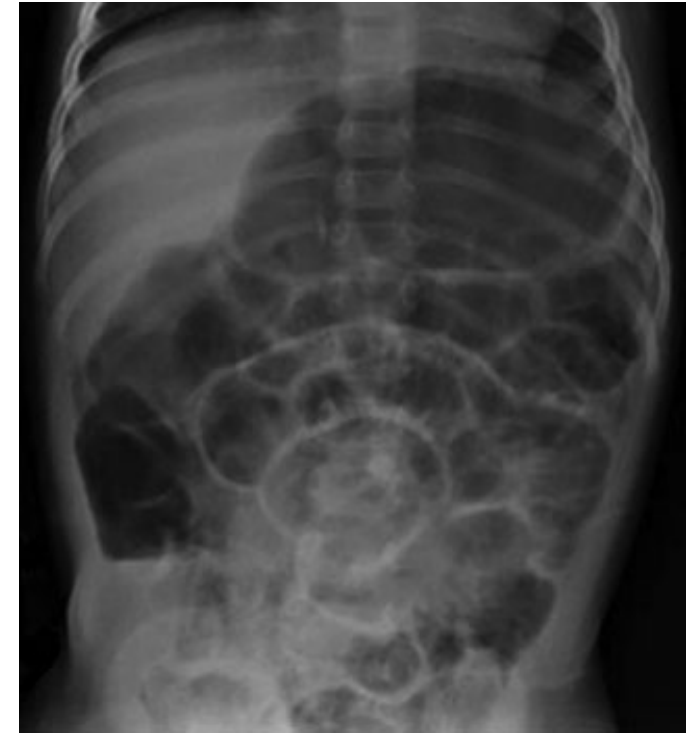

Fig. 2 Abdominal X-ray. Right lower quadrant fixed air fluid level and small bowel dilatation.

Several postoperative complications of primary nephrectomy following the NWTS protocol approach have been described, with bowel obstruction being the most common appearing in 5.1 to $6.9 \%$ of the patients, compared with $2.5 \%$ of the patients following the Societe Internationale D'oncologie Pediatrique protocol; postoperative adhesions followed by intussusceptions and internal hernias have all been described. ${ }^{3,14}$

Postoperative intussusception usually occurs within the first postoperative week with a mean of 5 days; ${ }^{15,16}$ bowel adhesions in infants and children causing bowel obstruction usually have a late presentation with $66 \%$ of them presenting 1 year after the initial procedure in some series, and only $6.8 \%$ of them being secondary to the resection of WT. ${ }^{17}$ Internal hernias following a nephrectomy with small bowel obstruction usually have an acute presentation and can be either transmesenteric or in the retroperitoneal fossa. ${ }^{14,18-20}$

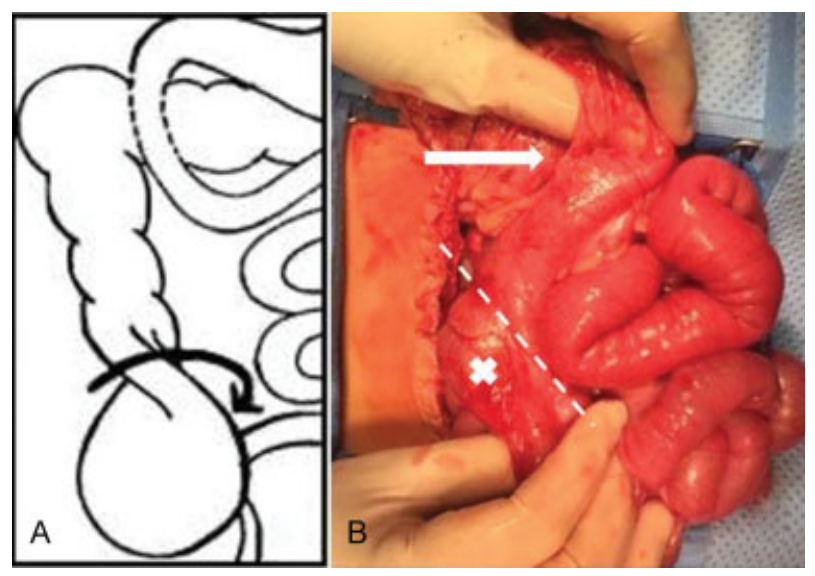

Fig. 3 (A) Scheme demonstrating the cecal volvulus found during reintervention. (B) Solid arrow showing medial mobilization of right colon. Dashed lined showing the transition point of obstruction after detorsion of the cecum (" $x$ " mark). 
An important differential diagnosis of patients presenting with volvulus is malrotation. This entity should always be kept in mind when assessing any infant or child with symptoms of vomiting and pain, particularly when the vomiting is bile stained. Ultrasound examination may be helpful but is not secure enough to exclude the diagnosis. Laparotomy or laparoscopy is the only way to be sure. ${ }^{21}$

From the several risk factors identified that can increase the rate of postoperative surgical complications following the resection of a WT (including higher local tumor stage, intravascular extension, and en bloc resection of other visceral organs ${ }^{3}$ ), none of them has been reported as a specific risk factor for postoperative bowel obstruction. ${ }^{22}$

A cecal volvulus following a right nephrectomy in adults has been described previously after releasing the cecum from the peritoneum and positioning it medially, $5,9,10$ although it has also been described following a left-sided nephrectomy. ${ }^{8}$

The following have been described as options for cecal volvulus management: cecopexy, simple detorsion without fixation, detorsion with cecopexy, tube cecostomy, and ileocolectomy. ${ }^{23}$ There is no consensus on which is the best option for the management of the cecal volvulus, but there is an agreement between series that the management should rely on the viability of the bowel. ${ }^{24-26}$ Detorsion might be sufficient for patients with a viable bowel, without gangrene, and a cecostomy has had a higher rate of reported complications in almost half of the series, with recurrence in approximately $15 \%$ and mortality rates of $20 \%{ }^{23}$

We decided to do a detorsion of the cecal volvulus because the bowel was viable; even though we do not usually close the lateral peritoneum following a nephrectomy for WT, we decided to close the lateral peritoneum on the second intervention as a safety measure to keep the colon in its place.

A volvulus of the cecum should be considered as a possible cause of intestinal obstruction in the postoperative period following a right nephrectomy for WT, with a surgical intervention being the treatment of choice. The management of a cecal volvulus should be decided based on the viability of the bowel. A simple detorsion might be sufficient for the successful management of the condition, but we believe that closing the lateral peritoneum helped minimize the movement of the right colon during our second intervention. Although based on the evidence we cannot conclude that closure of the lateral peritoneum is mandatory, we do believe that this might have played a huge role in the effectiveness of the procedure.

\section{Conflict of Interest}

None.

\section{Acknowledgment}

The author would like to acknowledge Dr Rodrigo GonzalezUrquijo and the Instituto Tecnologico y de Estudios Superiores de Monterrey, Medical Research Department.

\section{References}

1 Hamilton TE, Shamberger RC. Wilms tumor: recent advances in clinical care and biology. Semin Pediatr Surg 2012;21(01):15-20

2 Sudour-Bonnange H, Lervat C, Renaud F, Gauthier H, Rocourt N. An unusual late recurrence of Wilms tumor. J Pediatr Hematol Oncol 2016;38(04):e151-e153

3 Ritchey ML, Shamberger RC, Haase G, Horwitz J, Bergemann T, Breslow NE. Surgical complications after primary nephrectomy for Wilms' tumor: report from the National Wilms' Tumor Study Group. J Am Coll Surg 2001;192(01):63-68, quiz 146

4 Takada K, Hamada Y, Sato M, et al. Cecal volvulus in children with mental disability. Pediatr Surg Int 2007;23(10):1011-1014

5 Scott CD, Trotta BM, Dubose JJ, Ledesma E, Friel CM. A cruel twist: post-operative cecal volvulus. Ulus Travma Acil Cerrahi Derg 2008;14(02):158-162

6 Tannouri S, Hendi A, Gilje E, Grissom L, Katz D. Pediatric colonic volvulus: a single-institution experience and review. J Pediatr Surg 2017;52(06):1062-1066

7 Wilson H, Hardy JD, Farringer JLJr. Intestinal obstruction: causes and management in infants and childhood. Ann Surg 1955;141:777-788

8 Etheredge EE, Martz M, Anderson CB. Volvulus of the cecum following transplant donor nephrectomy. Surgery 1977;82(05):764-767

9 Ali Khan S, Desai PG, Siddharth P, Smith N. Volvulus of cecum following simple nephrectomy. Urol Int 1985;40(01):1-2

10 Eng M, Ravindra K. Cecal volvulus following laparoscopic nephrectomy and renal transplantation. JSLS 2009;13(04):612-615

11 Bhatnagar S. Management of Wilms' tumor: NWTS vs SIOP. J Indian Assoc Pediatr Surg 2009;14(01):6-14

12 Metzger ML, Dome JS. Current therapy for Wilms' tumor. Oncologist 2005;10(10):815-826

13 Boccon-Gibod L, Rey A, Sandstedt B, et al. Complete necrosis induced by preoperative chemotherapy in Wilms tumor as an indicator of low risk: report of the international society of paediatric oncology (SIOP) nephroblastoma trial and study 9. Med Pediatr Oncol 2000;34(03):183-190

14 Ritchey ML, Kelalis PP, Etzioni R, Breslow N, Shochat S, Haase GM. Small bowel obstruction after nephrectomy for Wilms' tumor. A report of the National Wilms' Tumor Study-3. Ann Surg 1993;218(05):654-659

15 de Vries S, Sleeboom C, Aronson DC. Postoperative intussusception in children. Br J Surg 1999;86(01):81-83

16 Velin P, Dupont D, Parizot P, Puig C. [Postoperative intestinal intussusception in children]. Ann Fr Anesth Reanim 1992;11(05):584-586

$17 \mathrm{Al}$-Salem $\mathrm{AH}$, Oquaish $\mathrm{M}$. Adhesive intestinal obstruction in infants and children: the place of conservative treatment. ISRN Surg 2011;2011(01):645104

18 Di Rocco G, Casella G, Giannotti D, et al. Transmesenteric hernia after right nephrectomy: diagnostic and therapeutic management. Eur Rev Med Pharmacol Sci 2013;17(06):842-844

19 Pielaciński K, Szczepanik AB, Wymysłowski P, Wójtowicz J. [Incarcerated hernia of the inferior ileocecal recess-a rare nephrectomy complication]. Wiad Lek 2012;65(03):203-205

20 Milosevic I, Dorion H, Ricchiuti D. Incarcerated retroperitoneal hernia following total extraperitoneal laparoscopic radical nephrectomy. JSLS 2011;15(03):424-426

21 Millar AJ, Rode H, Cywes S. Malrotation and volvulus in infancy and childhood. Semin Pediatr Surg 2003;12(04):229-236

22 Regan JP, Cho ES, Flowers JL. Small bowel obstruction after laparoscopic donor nephrectomy. Surg Endosc 2003;17(01):108-110

23 Rabinovici R, Simansky DA, Kaplan O, Mavor E, Manny J. Cecal volvulus. Dis Colon Rectum 1990;33(09):765-769

24 Madiba TE, Thomson SR. The management of cecal volvulus. Dis Colon Rectum 2002;45(02):264-267

25 Hjortrup A, Friis J, Merser S, Bredesen J. Volvulus of the cecum. Dan Med Bull 1987;34(06):331-333

26 Wright TP, Max MH. Cecal volvulus: review of 12 cases. South Med J 1988;81(10):1233-1235 